\title{
Study protocol for a parallel-group, double- blinded, randomized, controlled, noninferiority trial: the effect and safety of hybrid electroconvulsive therapy (Hybrid- ECT) compared with routine electroconvulsive therapy in patients with depression
}

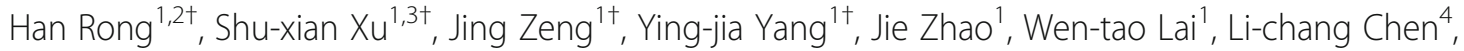
Wen-feng Deng ${ }^{5}$, Xiangyang Zhang ${ }^{6}$, Ying-li Zhang ${ }^{1}$, Min-zhi Li ${ }^{1}$, Li Xiao ${ }^{7}$ and Xin-hui Xie ${ }^{1,3,5^{*}}$ (D)

\begin{abstract}
Background: Electroconvulsive therapy (ECT) is the most rapid and effective treatment for patients with depression, ECT can achieve remarkable antidepressant effects in the initial 3-4 sessions, but significant side effects limit its use. However, recent low-charge electrotherapy (LCE) studies have demonstrated antidepressant or antipsychotic effects with significantly fewer side effects. The aim of this study is to propose a novel two-step charge set strategy for ECT treatment, referred to as Hybrid-ECT, to decrease side effects by using a low charge while preserving treatment efficacy.
\end{abstract}

Methods/design: A randomized, double-blinded, standard-controlled, parallel-group design will be carried out. We plan to enroll 112 inpatients diagnosed with depression (unipolar or bipolar) and randomly assign them to conventional ECT (control group) or to Hybrid-ECT (treatment group, 3 ECT sessions followed by LCE sessions (approximately 2.8 joules per session)). We will evaluate participants across a wide variety of domains including clinical symptoms, cognitive, psychological and functional metrics. We will also perform magnetic resonance imaging (MRI) and event-related potential (ERPs) assessments during treatment to explore brain function differences between ECT and LCE.

Discussion: This research proposes a simple but completely novel ECT strategy that aims to rapidly relieve depressive symptoms and minimize side effects. The mechanism of ECT and LCE will be further discussed.

Trial registration: Chinese Clinical Trial Registry, Number: ChiCTR1900022905 (Registration date: April 30, 2019).

Keywords: Study protocol, Randomized controlled trial, Hybrid electroconvulsive therapy, Electroconvulsive therapy, Depression

\footnotetext{
*Correspondence: xxh.med@gmail.com; xin-hui.xie@live.com

${ }^{\dagger} H a n$ Rong, Shu-xian Xu, Jing Zeng and Ying-jia Yang contributed equally to this work.

'Department of Psychiatry, Shenzhen Kangning Hospital, Shenzhen,

Guangdong, China

${ }^{3}$ Center of Acute Psychiatry Service, Second People's Hospital of Huizhou,

Huizhou, Guangdong, China

Full list of author information is available at the end of the article
}

(c) The Author(s). 2019 Open Access This article is distributed under the terms of the Creative Commons Attribution 4.0 International License (http://creativecommons.org/licenses/by/4.0/), which permits unrestricted use, distribution, and reproduction in any medium, provided you give appropriate credit to the original author(s) and the source, provide a link to the Creative Commons license, and indicate if changes were made. The Creative Commons Public Domain Dedication waiver (http://creativecommons.org/publicdomain/zero/1.0/) applies to the data made available in this article, unless otherwise stated. 


\section{Background}

Globally, major depressive disorder (MDD) is one of the most common psychiatric disorders, rated as one of the five leading cause of years lived with disability (YLDs) in 2016 and associated with substantial disabilities [1]. Patients with depression often show high suicide risk [2], severe impairments in social function [3] and cognitive dysfunction [4]. According to the 1995-1999 mortality data provided by the Minister of Health of the People's Republic of China, the mean annual suicide rate was 23 per 100000 , and there were a total of 287000 suicide deaths per year [5]. Therefore, it is especially important to quickly and effectively treat depression patients.

Electroconvulsive therapy (ECT) administered with anesthesia and neuromuscular blockers is the most effective treatment for depression [6-9]. However, the use of ECT is impeded by adverse effects (AEs), such as acute headache, dizziness and confusion [10], especially the cognitive function impairments, which may be affected by the stimulus intensity, number of treatments or electrode placement $[11,12]$. Thus, researchers have been working on methods to quickly relieve symptoms while minimizing side effects.

One important feature of ECT is the rapid antidepressant effect in the early stage of treatment. Kellner et al. conducted a double-blind, controlled trial to compare the efficacy and cognitive effects of three electrode placement methods in ECT [13]. The results demonstrated that all placements resulted in rapid remission over the early course of treatment; during the first 3-4 ECT sessions, the bitemporal placement resulted in a more rapid decrease in symptom ratings than the other placements, followed by small changes in symptom ratings in the subsequent sessions for all placement groups. Some articles have reported that patients could go into remission after the first ECT session [14-16]. The findings indicated that the decline in symptom rating of later stage was not as rapid as the early period; furthermore, as the number of ECT sessions increased, the side effects became more pronounced [17], which suggests that we should change the mode of ECT procedure.

At present, based on existing ECT knowledge, a charge-induced seizure is considered to be a key feature. However, after reviewing the literature, we found an interesting phenomenon. Some ECTs that failed to induce seizures also demonstrated antidepressant effects but without severe side effects [18-20]. The safety and efficacy of low-charge nonconvulsive electrotherapy (NET) for MDD was first studied in detail by Regenold et al. in 2015 [21]. The authors demonstrated that the therapeutic effect of NET in patients with resistant MDD was similar to that of ECT, while serious AEs were not observed. Although the study was small, unblinded, nonrandomized and uncontrolled by a sham or standard procedure, and the results were susceptible to both investigator and subject bias, they exhibited the potential of low-charge electrotherapy (LCE) for mental diseases and raised the following question: are high charges or seizures essential? Subsequently, a double-blind, randomized, controlled pilot clinical trial with schizophrenia patients was conducted by our team; the results suggested that LCE (2.8 joules per session) without seizures exerted similar antipsychotic effects while causing fewer AEs than ECT [22]. Although the sample sizes have been small, LCE has the potential to be a safe and effective treatment for patients with schizophrenia or depression. However, our inhouse data (detailed information please see Additional file 3: Table S2 and Additional file 4: Figure S1) revealed that LCE relieved depression more slowly in the first few sessions than ECT.

Considering that patients with depression need quick symptom relief with few side effects, we propose a new two-step charge set strategy for ECT treatment, referred to as Hybrid-ECT. In Hybrid-ECT, conventional ECT is conducted during the first 3 sessions (the first step) because according to Kellner's research, the Hamilton Rating Scale for Depression (HAMD-24) score reduction trajectory is steep during the first 3 ECT sessions (an approximation based on Figure 2 of Kellner's article [13]), and then LCE is conducted until the treatment is terminated (the second step). We hope that the two-step Hybrid-ECT could combine ECT's strength of an early rapid antidepressant effect and LCE's advantage of fewer side effects. Therefore, our hypotheses were as follows: 1) Hybrid-ECT exerts a noninferior antidepressant effect relative to ECT over the whole course of the treatment; and 2) compared with the routine ECT charge set strategy, Hybrid-ECT may cause fewer AEs, especially in the later part of treatment (i.e., after 3 ECT sessions). To test these hypotheses, we designed the present randomized, double-blinded, parallel-group, controlled clinical trial. Since the intervention arm of this trial is a change in the current ECT charge setting strategy, routine ECT was selected as the standard-control group; and the trial is designed as a noninferiority study on the antidepressant effects of Hybrid-ECT and ECT.

\section{Methods/design Study design}

This clinical trial is a randomized, double-blinded, parallelgroup, standard-controlled, noninferiority study to detect the efficacy and side effects of a novel ECT charge setting strategy called Hybrid-ECT for the treatment of patients with depression compared to routine ECT. Participants, neuropsychological measurement raters, magnetic resonance imaging (MRI) and event-related potential (ERP) operators, patient psychiatrists, nurses, and researchers will be blinded to the patient treatment assignment. After each ECT/LCE procedure, the ECT operator will reset the 
energy parameters to prevent others from detecting the participant's group. The study flow chart is shown in Fig. 1.

\section{Study population and recruitment}

A total of 112 inpatients with unipolar or bipolar depression will be recruited by psychiatrists in Shenzhen Kangning Hospital (Mental Health Center of Shenzhen city, which has more than 1000 beds) in Shenzhen city, Guangdong Province, PR China.

\section{Inclusion criteria}

1. Patient $(18<$ age $<60)$ accepted ECT for treatment because of severe depression and on a stable psychotropic medication regime more than 2 weeks;

2. Patient met the following ICD-10 criteria [23] for diagnosis of unipolar or bipolar depression (F31.4, F31.5, F32, F33);

3. Patient had an HAMD-24 score $\geq 21$;

4. Patient was competent to provide informed consent; and.

5. Patient was sufficiently fluent in Chinese to ensure valid responses to the neuropsychological tests.

\section{Exclusion criteria}

1. Failure to respond to earlier ECT;

2. ECT within the last 6 months;

3. Rapid-cycling or mixed-episode bipolar disorder;
4. Medication use incompatible with ECT treatment (such as lithium, benzodiazepine, antiepileptic drugs, etc.). Use of these drugs will be stopped at least 5 half-lives before the start of treatment;

5. Inability to follow the study protocol;

6. Unstable, serious comorbid medical condition (Parkinson's disease, multiple sclerosis, stroke, alcohol use disorder, etc.) or history of epilepsy;

7. Intelligence quotient (IQ) $\leq 70$ tested by the Wechsler Adult Intelligence Scale 4th edition [24];

8. $\leq 9$ years of education;

9. Pregnancy or women without adequate contraception; and.

10. Young Mania Rating Scale (YMRS) score [25] $\geq 20$.

\section{Withdrawal criteria}

The patient will be withdrawn and unblinded at any time if the exclusion criteria are met, the psychiatrist finds that the patient could be better served with other treatments, there is a decrease in the HAMD-24 score of $<3$ points between the latest two visits, or the patient decides to withdraw. When a patient withdraws his or her consent, the reason and withdrawal date will be recorded.

\section{Interventions}

ECT group (control group)

Anesthesia Patients will be administered etomidate for anesthesia, succinylcholine as a muscle relaxant, and

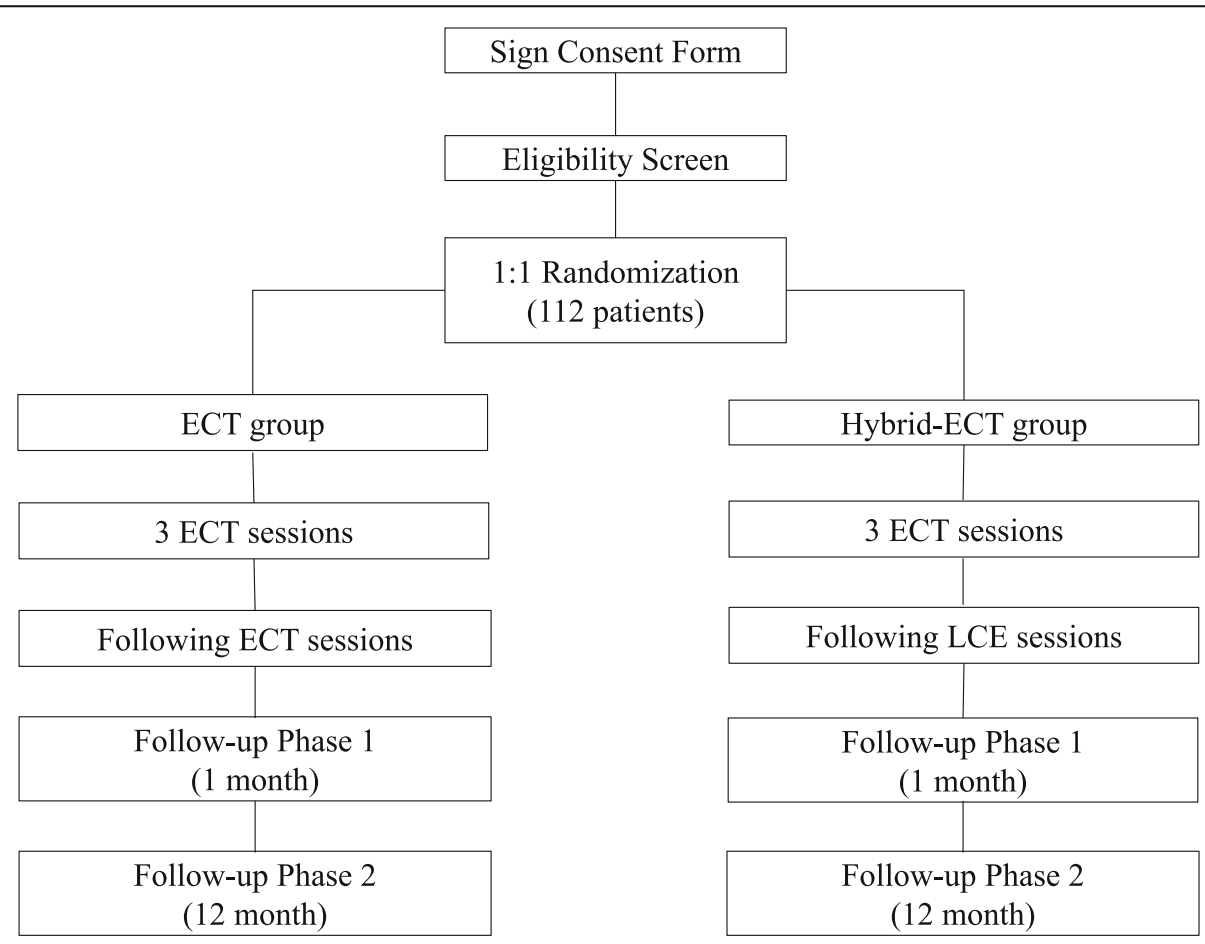

Fig. 1 Study flow chart 
atropine for the suppression of gland secretions, and pure oxygen will be supplied during each treatment session.

Placement of stimulation electrodes Because bitemporal electrode placement achieved a more rapid and earlier decrease in symptom ratings [13], bitemporal electrode placement will be used on each patient. For the bitemporal electrode placement, the two electrodes are applied $2-3 \mathrm{~cm}$ above the midpoint of the line connecting the outer canthus of the eye and the external auditory meatus on each side of the head [13].

Stimulus and seizure threshold (ST) titration The ECT or LCE procedure will be administered with a spECTrum 5000Q ECT instrument (MECTA Corporation, OR, USA) three times a week. The pulse width is set to $1 \mathrm{~ms}$, and the current is set to $800 \mathrm{~mA}$. The ST titration procedure basically follows Mankad et al. [12]. The energy set of the first ECT session uses the titration procedure described in the Additional file 1: Table S1. If the seizure induction is unsuccessful or the seizure lasts less than $15 \mathrm{~s}$ (based on electroencephalogram (EEG)), the patient will be restimulated at a higher energy level after a short delay. The restimulation process could be repeated up to three times during the first treatment. If all four stimulations fail to induce an adequate seizure, the dose set for the titration in the next session will be set at 2 steps higher than the last stimulation dose. The ECT charge dose for subsequent treatments is set as follows: 1.5 * ST, similar to Kellner et al. [13].

Seizure adequacy Seizure detection is performed using a single channel EEG from the ECT instrument. The seizure duration recorded by EEG is checked by a certified electroencephalographer who is blinded to the participant's status.

\section{Hybrid-ECT group (treatment group)}

Anesthesia Same as the ECT group.

Placement of stimulation electrodes Same as the ECT group.

Stimulus The energy set of the first three sessions is the same as that in the ECT group; the subsequent sessions use LCE, and the energy is set to the minimum $(2.8 \mathrm{~J})$ with a stimulus duration $=0.5 \mathrm{~s}$ and a frequency $=20 \mathrm{~Hz}$, as we have previously reported [22].

Seizure adequacy Same as the ECT group.

Follow-up scheme To evaluate the acute and chronic influence of ECT and Hybrid-ECT respectively, we set a two-stage follow-up schedule (see Table 1). The first follow-up phase is set at the one-month after the last ECT/LCE session, and the second phase is a one-year follow-up following the first phase (see Table 1).

Randomization After receiving detailed information about this trial, signing the consent form, passing the eligibility screen and completing the baseline test, the enrolled patients will be assigned a sequential patient number. If a patient discontinues from the study between the randomization and the first ECT procedure, the patient will be excluded from the final analyses, and he or she will not be allowed to rejoin the study.

A statistician with no other connection to the trial will generate the randomization sequence using the default random number generator of SAS version 9.4 (SAS Institute Inc., Cary, NC, USA). The randomization sequence list will remain concealed from the investigators. A research nurse who will not participate in the clinical evaluation will assign the participants to their group.

Participants, neuropsychological measurement raters, MRI and ERP operators, patient psychiatrists, nurses, and researchers will be blinded to the patient treatment assignment. If unblinding is determined to be necessary, the investigators must report all unblinding (with reason) on the corresponding case report form (CRF).

\section{Assessment of the effectiveness of the blind methods} Participants and raters will be asked to which group the participant had been allocated, and about the strength of confidence of their guess as well as the reasons for their guess at every visit after the randomization. The ratios of right guesses/wrong guesses will be used to assess the effectiveness of the blinding procedure.

Medication during ECT/hybrid-ECT sessions Patients in both arms will be maintained on their previously prescribed antidepressants and antipsychotics (usually used for patients with bipolar depression and patients with depression and psychotic symptoms) during the trial. Anticonvulsant drugs, lithium, or mood stabilizers will be discontinued during the course of ECT/Hybrid-ECT treatment. Patients exhibiting agitation or feeling anxious could be temporarily administered short half-life benzodiazepines, but the administration of benzodiazepines will be prohibited $24 \mathrm{~h}$ before the ECT/LCE session. When patients suffer from insomnia, zopiclone, eszopiclone, or zolpidem can be temporarily given to help them sleep.

Medication during the follow-up phase Medication during the follow-up period is essentially the same as that during the ECT/Hybrid-ECT phase; however, lithium, mood stabilizers, or antiepileptic drugs are allowed. The detailed and individualized pharmacological plan will be determined by the patient's psychiatrist. 
Table 1 Visit procedure

\begin{tabular}{|c|c|c|c|c|c|c|c|c|c|}
\hline \multirow[b]{2}{*}{ Time point } & \multirow{2}{*}{$\begin{array}{l}\text { Enrollment } \\
-t_{1}\end{array}$} & \multirow{2}{*}{\begin{tabular}{|l} 
Allocation \\
$t_{0}$ \\
\end{tabular}} & \multicolumn{5}{|c|}{ ECT/Hybrid-ECT treatment Phase } & \multirow{2}{*}{$\begin{array}{l}\text { Follow-up Phase } 1 \\
\mathrm{t}_{6}\end{array}$} & \multirow{2}{*}{$\begin{array}{l}\text { Follow-up Phase } 2 \\
\mathrm{t}_{7}-\mathrm{t}_{10}\end{array}$} \\
\hline & & & $\mathrm{t}_{1}$ & $t_{2}$ & $t_{3}$ & $t_{4}$ & $t_{5}$ & & \\
\hline Diagnostic interview & $\mathbf{x}$ & & & & & & & & \\
\hline Informed consent & $\mathbf{x}$ & & & & & & & & \\
\hline MINI & $\mathbf{x}$ & & & & & & & & \\
\hline $\begin{array}{l}\text { Inclusion/exclusion } \\
\text { criteria }\end{array}$ & $\mathbf{x}$ & & & & & & & & \\
\hline \multicolumn{10}{|l|}{ Interventions } \\
\hline ECT arm & & & \multicolumn{5}{|l|}{ ECT } & & \\
\hline Hybrid-ECT arm & & & $\stackrel{\text { ECT }}{\longrightarrow}$ & \multicolumn{4}{|r|}{ 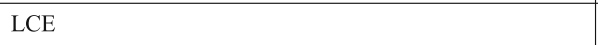 } & & \\
\hline $\begin{array}{l}\text { Post ECT/LCE } \\
\text { number }\end{array}$ & & & 3 & 6 & 9 & $\begin{array}{l}12 \text { or last } \\
\text { session }\end{array}$ & $\begin{array}{l}\text { One week post the last } \\
\text { ECT/LCE session }\end{array}$ & 1 month & $\begin{array}{l}4-, 7-, 10-, 13- \\
\text { month }\end{array}$ \\
\hline \multicolumn{10}{|l|}{ Clinical assessment } \\
\hline HAMD-24 & $\mathbf{x}$ & & $\mathbf{x}$ & $\mathbf{x}$ & $\mathbf{x}$ & $\mathbf{x}$ & $\mathbf{x}$ & $\mathbf{x}$ & $\mathbf{x}$ \\
\hline HAMA & $\mathbf{x}$ & & $\mathbf{x}$ & $\mathbf{x}$ & $\mathbf{x}$ & $\mathbf{x}$ & $\mathbf{x}$ & $\mathbf{x}$ & $\mathbf{x}$ \\
\hline YMRS & $\mathbf{x}$ & & $\mathbf{x}$ & $\mathbf{x}$ & $\mathbf{x}$ & $\mathbf{x}$ & $\mathbf{x}$ & $\mathbf{x}$ & $\mathbf{x}$ \\
\hline CGI & $\mathbf{x}$ & & $\mathbf{x}$ & $\mathbf{x}$ & $\mathbf{x}$ & $\mathbf{x}$ & $\mathbf{x}$ & $\mathbf{x}$ & $\mathbf{x}$ \\
\hline PANSS & $\mathbf{x}$ & & $\mathbf{x}$ & $\mathbf{x}$ & $\mathbf{x}$ & $\mathbf{x}$ & $\mathbf{x}$ & $\mathbf{x}$ & $\mathbf{x}$ \\
\hline SF-36 & $\mathbf{x}$ & & & & & & & $\mathbf{x}$ & $\mathbf{x}$ \\
\hline SDS & $\mathbf{x}$ & & $\mathbf{x}$ & $\mathbf{x}$ & $\mathbf{x}$ & $\mathbf{x}$ & $\mathbf{x}$ & $\mathbf{x}$ & $\mathbf{x}$ \\
\hline SAS & $\mathbf{x}$ & & $\mathbf{x}$ & $\mathbf{x}$ & $\mathbf{x}$ & $\mathbf{x}$ & $\mathbf{x}$ & $\mathbf{x}$ & $\mathbf{x}$ \\
\hline EMQ & $\mathbf{x}$ & & $\mathbf{x}$ & $\mathbf{x}$ & $\mathbf{x}$ & $\mathbf{x}$ & $\mathbf{x}$ & $\mathbf{x}$ & $\mathbf{x}$ \\
\hline \multicolumn{10}{|c|}{ Neuropsychological assessment } \\
\hline WAIS & \begin{tabular}{|l|}
$\mathbf{x}$ \\
\end{tabular} & & & & & & & & \\
\hline MMSE & $\mathbf{x}$ & & $\mathbf{x}$ & & & & $\mathbf{x}$ & $\mathbf{x}$ & $\mathbf{x}$ \\
\hline RBANS & $\mathbf{x}$ & & $\mathbf{x}$ & & & & $\mathbf{x}$ & $\mathbf{x}$ & $\mathbf{x}$ \\
\hline Digit Symbol Test & $\mathbf{x}$ & & $\mathbf{x}$ & & & & $\mathbf{x}$ & $\mathbf{x}$ & $\mathbf{x}$ \\
\hline Stroop Test & $\mathbf{x}$ & & $\mathbf{x}$ & & & & $\mathbf{x}$ & $\mathbf{x}$ & $\mathbf{x}$ \\
\hline Trail Making Test & $\mathbf{x}$ & & $\mathbf{x}$ & & & & $\mathbf{x}$ & $\mathbf{x}$ & $\mathbf{x}$ \\
\hline ORT $^{\mathrm{a}}$ & $\mathbf{x}$ & & $\mathbf{x}$ & $\mathbf{x}$ & $\mathbf{x}$ & $\mathbf{x}$ & $\mathbf{x}$ & $\mathbf{x}$ & $\mathbf{x}$ \\
\hline Lab test & $\mathbf{x}$ & & $\mathbf{x}$ & & & & $\mathbf{x}$ & $\mathbf{x}$ & \\
\hline MRI acquisition & $\mathbf{x}$ & & $\mathbf{x}$ & & & & $\mathbf{x}$ & & \\
\hline ERP examination & $\mathbf{x}$ & & $\mathbf{x}$ & & & & $\mathbf{x}$ & & \\
\hline
\end{tabular}

a: test after every ECT/LCE session

HAMD-24: Hamilton depression rating scale, HAMA Hamilton anxiety rating scale, CGI Clinical global impressions, YMRS Young mania rating scale, PANSS Positive and negative syndrome scale, SF-36 MOS 36-item short-form health survey, SDS Self-rating depression scale, SAS Self-rating anxiety scale, EMQ Everyday memory questionnaire, WAIS Wechsler adult intelligence scale 4th edition, MMSE Mini-mental state examination, RBANS Repeatable battery for the assessment of neuropsychological status, ORT Orientation recovery test

\section{Outcome measures}

\section{Visit procedure}

Patients will be interviewed during twelve visits. The detailed visit procedure is shown in Table 1.

\section{Primary outcome measure}

The primary outcome measure is the change in total HAMD-24 score at endpoint (visit $t_{5}$ ) from baseline.

\section{Secondary outcome measures}

The secondary outcome measures include the response rate (defined as a HAMD-24 score that shows a more than $50 \%$ reduction from the baseline) and remission rate (remission is defined as HAMD-24 score $\leq 10)$ at $t_{5}$ and the scores on the Clinical Global Impressions (CGI) scale [26], Positive and Negative Syndrome Scale (PANSS) [27], MOS 36-item Short-form Health Survey (SF-36) [28], Hamilton Anxiety Rating Scale (HAMA) [29], YMRS [25], Self-Rating Depression Scale (SDS) [30], Self-Rating Anxiety Scale (SAS) [31], and Everyday Memory Questionnaire (EMQ) [32].

\section{Neuropsychological assessment}

A comprehensive neurocognitive battery will be performed, as presented in Table 1. Neuropsychological evaluations will be carried out by trained psychologists who will be blinded to the patient groups. The cognitive domains to be tested include general intelligence, global status, attention, processing speed, language, visuospatial 
function, executive function and memory. IQ will be assessed by the Wechsler Adult Intelligence Scale 4th edition [24]. A modified version of the Mini-Mental State Examination (MMSE) [33] will be used to test global status. The Repeatable Battery for the Assessment of Neuropsychological Status (RBANS) is designed to assess attention, language, visuospatial, immediate memory and delayed memory [34]. Processing speed will be assessed by the digit symbol test [35]. Stroop reaction time, Stroop color-word test [36] and trail making test (A, B) [37] will be used for examination of executive function. Orientation recovery is assessed at every ECT/ LCE session. The time to orientation test is assessed with continuous questioning on five items (name, place, day, age, date of birth) for $90 \mathrm{~min}$ [38]. A correct response on four of five items is required. Patients who do not meet the criteria for recovery are assigned scores of 100 min. The subjective cognitive evaluation will be evaluated by the Squire Subjective Memory Questionnaire [39].

\section{$M R I$ and ERP acquisition}

ECT can cause changes in brain function [40], metabolism [41], and structure [42], especially in hippocampal volume [43], which may be related to neurogenesis in the hippocampal dentate gyrus, so the aims of our MRI and ERP are as following: 1) use the MRI to observe the changes of hippocampus in volume before and after treatment and between the two groups; 2) use the fMRI and DTI to observe ECT/LCE-related activation, functional connections, and changes in nerve fiber connections; 3) use MRS to observe metabolic changes of hippocampus before and after ECT; 4) use ERP to observe the EEG changes related to ECT/LCE.

\section{MRI protocol}

MRI will be performed at 3 time points: $t_{0}(\sim 24-48 \mathrm{~h}$ before baseline), $t_{1}(\sim 24-48 \mathrm{~h}$ after the three first ECT and before the fourth ECT/LCE) and $t_{5}(\sim 7$ days the last of ECT/LCE). All participants will be scanned at the Shenzhen Kangning Hospital Neuropsychiatry Imaging Center in a 3 T Discovery MR750 scanner (GE Healthcare, Milwaukee, USA) equipped with an eight-channel head coil. A standard MRI protocol will be applied at each time point, including a T1-weighted spoiled gradient echo, SPGR (TE = min full; $\mathrm{TR}=6.7 \mathrm{~ms}$; flip angle = $12^{\circ}$; field of view $=256 \mathrm{~mm}$; voxel size $=1.0 * 1.0 * 1.0$ $\mathrm{mm}^{3}$; matrix $=256 * 256$ ); a T2-weighted high resolution hippocampus sequence $(\mathrm{TE}=50 \mathrm{~ms}$; $\mathrm{TR}=8020.0 \mathrm{~ms}$; flip angle $=122^{\circ}$; field of view $=175 \mathrm{~mm}$; voxel size $=0.4 * 0.4$ * $2.0 \mathrm{~mm}^{3}$; matrix $=448 * 448$ ); a resting-state fMRI scan with a BOLD sequence $(\mathrm{TE}=25 \mathrm{~ms}$; $\mathrm{TR}=2000 \mathrm{~ms}$; field of view $=220 \mathrm{~mm}$; voxel size $=3.4 * 3.4 * 3.2 \mathrm{~mm}^{3}$; matrix $=64 * 64$; volume number $=240$ ); a DTI sequence $(\mathrm{TE}=81.4 \mathrm{~ms} ; \mathrm{TR}=8724.0 \mathrm{~ms} ;$ field of view $=224 \mathrm{~mm}$; voxel size $=2.0 * 2.0 * 2.0 \mathrm{~mm}^{3}$; matrix $=112 * 112$; number of diffusion directions $=64 ; \mathrm{b}$ values $=0,1000 \mathrm{~s}$ / $\mathrm{mm}^{2}$ ); ${ }^{1} \mathrm{H}$ - magnetic resonance spectroscopy (MRS) sequences for the ROIs including the hippocampus, thalamus, anterior cingulate and posterior cingulate $(\mathrm{TE}=$ $35.0 \mathrm{~ms}$; TR $=1500 \mathrm{~ms}$ ) [44-46]; and a 3D ASL sequence $(\mathrm{TE}=10.5 \mathrm{~ms} ; \mathrm{TR}=4389 \mathrm{~ms} ;$ post-labeling delay $=1025$ $\mathrm{ms}$; field of view $=240 \mathrm{~mm}$ ).

\section{ERP measures}

The ERP data will be recorded using BrainAmp Amplifier (Brain Products GmbH, Zeppelinstraße 782,205 Gilching, Germany), and electrodes will be placed at 32 scalp locations based on the 10-20 system [47]. An electrode linked ears/mastoid reference scheme will be used. The electrode impedance will be kept below $10 \mathrm{~K} \Omega$. Amplifiers will have a bandpass of $0.1-50 \mathrm{~Hz}$, and the stimulus rate will be 0.5 Hz. Continuous EEG records, at a sampling rate of 1000 $\mathrm{Hz}$, will be stored for further off-line analysis. In the ERP experiment, the patients will be told to stay relaxed and quiet. P300 and P50 paradigms and 3-min resting-state EEG recording with eyes closed will be selected for the measurement index of cognitive function. The classic auditory oddball paradigm will be used to measure P300. The classic P300 task will include a total of 200 target and standard stimuli, and the 40 target stimuli will be a tone at $60 \mathrm{~dB}$ of low frequency $(20 \%)$ and high pitch $(2000 \mathrm{~Hz})$, and the 160 standard stimuli will be a tone at $80 \mathrm{~dB}$ of high frequency $(80 \%)$ and low pitch $(1000 \mathrm{~Hz})$. The two stimuli presentations last $20 \mathrm{~ms}$. The participant will need to click a button as soon as they hear the stimuli to judge whether a tone was a standard stimulus or target stimulus $[48,49]$. P50 will be examined using conditioning (S1)testing (S2) paired acoustic stimulation with short wavelength and sound pressure set at $105 \mathrm{~dB}$. The first click produces an excitatory response and activates inhibitory pathways, and the response to the second click is normally suppressed. The pairs of clicks will be presented $500 \mathrm{~ms}$ apart and the interval between two paired stimuli is $10 \mathrm{~s}$ $[50,51]$. P50 is measured by the P50 ratio, which will be calculated as the S2 amplitude divided by the S1 amplitude. A P50 ratio closer to 0 is indicative of robust suppression (gating), whereas a ratio closer to 1 is indicative of diminished sensory gating [52]. The ERP procedures will be performed according to the MRI schedule.

\section{Safety assessment and AEs}

Safety data will include clinical examinations, blood tests, electrocardiogram (ECG), urine tests, and cognitive function assessments.

\section{AEs}

Any untoward medical occurrence during ECT/HybridECT treatment, which may or may not be causally linked 
with the ECT/LCE treatment, will be defined as an AE. All AEs will be recorded in the CRF. An important focus of this trial is to explore whether there are fewer side effects from Hybrid-ECT than from routine ECT, in addition to comparing total $\mathrm{AE}$ numbers and severity; therefore, all AEs will be categorized according to when they occurred (initial three ECT sessions or the subsequent ECT/LCE sessions) for further analyses.

\section{Sample size and power calculation}

Only one study [21] has measured changes in depression symptom and cognitive effects between pre- and postNET. Therefore, to test the first hypothesis, we used noninferiority tests [53] calculated using the Power Analysis and Sample Size Software (ver.15.0) (NCSS, LLC. Kaysville, Utah, USA) for the difference between two means (noninferiority margin $=4.0$, standard deviation $(\mathrm{SD})=8.0$, power $=0.8, \mathrm{a}=0.05$ (one-tailed), drop-out rate $=10 \%$ ) of the change in HAMD-24 scores with a 1:1 allocation, and we obtained a total sample size of 112 (56 for each arm).

\section{Statistical analyses \\ Neuropsychological and clinical outcomes}

Demographic and baseline data will be analyzed using independent two-sample $t$-tests or chi-square tests (or Fisher's exact tests). All analyses of clinical outcomes, neuropsychological results and safety indicators will be performed for the modified intent-to-treat population (MITTP) (at least one treatment and at least one postbaseline measure). We will use mixed-effects model repeated measures (MMRM) as the main analysis for the comparison of the primary and continuous secondary outcome measures. We will use chi-square/Fisher's exact tests to analyze the categorical outcome measures. We will use a "tipping point" analysis to assess the robustness of the primary analysis (MMRM) to possible deviations from the missing at random (MAR) assumption. The significance level will be set to 0.05 (two tailed). All statistical analyses will be performed with SAS (ver. 9.4) software (SAS Institute Inc., Cary, NC, USA). MRI and ERP data analyses will be performed by using the relevant professional software packages (FreeSurfer [54], FSL [55], EEGLAB [56], etc.).

\section{Discussion}

\section{Summary}

To the best of our knowledge, this is a completely novel energy set strategy for ECT procedures. We speculate that Hybrid-ECT may rapidly relieve depressive symptoms during the early stage, then we will convert to LCE until the symptoms are completely relieved, and the side effects will occur less often than those in the control group (routine ECT). Furthermore, we will also perform the MRI and ERP assessments before and after the ECT treatment to test the hypothesis that Hybrid-ECT and routine ECT share the same antidepressant mechanisms. The strengths of the study include an innovative experimental design, time-intensive visits, detailed neuropsychological assessments, and MRI and ERP examinations to accurately document the differences in efficacy and side effects between Hybrid-ECT and ECT.

\section{Limitations}

Potential weaknesses include a relatively small sample size and the expected heterogeneity in the patients' use of medications, both of which may increase the variance in the study measures. Furthermore, we lack a third arm using a sham ECT to further assess the potential therapeutic efficacy of Hybrid-ECT, but this task has proven difficult to accomplish due to the ethical concerns of exposing patients to anesthesia when there is no expectation of a clinical benefit. Therefore, we chose routine ECT as the standard control. A larger multi-center trial is required, along with methods for measuring biomarkers to better understand and regulate the application of Hybrid-ECT in the treatment of depression.

\section{Study feasibility}

Shenzhen Kangning Hospital is the largest psychiatric hospital in Shenzhen city, treating thousands of patients with depression every year. Our hospital has been conducting ECT for many years without serious medical accidents. The researchers have rich clinical and scientific experience, and we have published a relevant pilot clinical trial [22], which led us to design Hybrid-ECT to further reveal the secrets of ECT.

\section{Perspectives}

If Hybrid-ECT is found to rapidly relieve depressive symptoms with fewer side effects, this could have important implications for future ECT strategies in treating patients with depression and the scientific understanding of the mechanisms of ECT-related cognitive decline in patients. In addition, this study may improve the understanding of ECT-related antidepressant mechanisms.

\section{Trial status and dissemination}

Participant enrollment will be initiated approximately in July 2019 and is expected to be completed by July 2020. The findings of this study will be published in peer-reviewed scientific journals and presented at scientific conferences. The SPIRIT-Checklist please see the Additional file 2.

\section{Supplementary information}

Supplementary information accompanies this paper at https://doi.org/10. 1186/s12888-019-2320-3.

Additional file 1: Table S1. Charge titration procedure.

Additional file 2. SPIRIT-Checklist.

Additional file 3: Table S2. Results of preliminary trial. 
Additional file 4: Figure S1. The HAMD Change of preliminary trial.

\section{Abbreviations}

AE: Adverse effects; CGI: Clinical Global Impressions; CRF: Case report form; DMC: Data monitoring committee; ECT: Electroconvulsive therapy; EEG: Electroencephalogram; EMQ: Everyday Memory Questionnaire; ERPs: Event-related potentials; GCP: Good clinical practice; HAMA: Hamilton Anxiety Rating Scale; HAMD-24: Hamilton Rating Scale for Depression; Hybrid-ECT: Hybrid electroconvulsive therapy; IQ: Intelligence quotient; J: Joule; LCE: Low-charge electrotherapy; MAR: Missing at random; MDD: Major depressive disorder; MITTP: Modified intent-to-treat population MMRM: Mixed-effects model repeated measures; MMSE: Mini-Mental State Examination; MRI: Magnetic resonance imaging; MRS: Magnetic resonance spectroscopy; NET: Nonconvulsive electrotherapy; PANSS: Positive and Negative Syndrome Scale; RBANS: Repeatable Battery for the Assessment of Neuropsychological Status; SAS: Self-Rating Anxiety Scale; SD: Standard deviation; SDS: Self-Rating Depression Scale; SF-36: MOS 36-item Short-form Health Survey; ST: Seizure threshold; YMRS: Young Mania Rating Scale

\section{Acknowledgments}

We thank Dr. Jia Li and Ms. Jing-ya Zhang from Brain Function and Psychosomatic Medicine Institute, Second People's Hospital of Huizhou for their assistance in manuscript revision

\section{Authors' contributions}

X-hX conceived the concept of Hybrid-ECT. X-hX, HR and S-XX initiated the study design, and $Y$-jY, JZ and $W$-tL helped with implementation. HR and $X-$ $h X$ will supervise the whole trial. S-XX, X-hX, JZ, Y-jY, L-cC, W-tL, JZ and W-fD wrote the first draft. W-tL wrote the MRI protocol, JZ wrote the ERP protocol, W-fD and L-cC wrote the statistics protocol, and XyZ, M-ZL, Y-IZ, and LX contributed to critical revisions of the manuscript. All authors participated in the design of this trial and approved the final manuscript.

\section{Funding}

This work was supported by the Sanming Project of Medicine in Shenzhen (Grant Number: SZSM201812052), Science and Technology Program of Huizhou (Grant Number: 2018Y128), Science and Technology Program of Shenzhen (Grant Numbers: JCYJ20160429190927063 and JCYJ20170413101017457), Chinese National Natural Science Foundation (Grant Number: 81201047), and CAS Pioneer Hundred Talents Program (Grant Number: 2017-074). This work has not received funding/assistance from any commercial organizations. The funding sources had no roles in the design of this study and will not have any roles during the execution, analyses, interpretation of the data, or decision to submit results.

\section{Availability of data and materials}

A staff member will be appointed as a data administrator. Patients' data include electronic files and paper documents. The paper document will be stored in locked cabinets. The electronic files will be back up on the computers and be password and firewall protected. A data monitoring committee (DMC) within the good clinical practice (GCP) office in the Kangning Hospital has been established. The DMC is independent of the present trial staff and has the right to access and audit any data during the trial. The investigators and statistician will allow access to the final trial dataset after the trial is finished, and the participants will have access to his or her own data. As the sample size is moderate (112 patients), interim analyses will not be performed. The blood and stool samples and the MRI and ERP data will be stored for future use in ancillary studies. We will use a data-driven algorithm or machine learning algorithm to further detect the relationships between clinical outcomes and biological measures. The clinical data without personal information will be shared on the registration center after the trial finished.

\section{Ethics approval and consent to participate}

The study will be conducted in accordance with the Declaration of Helsinki and was approved by the ethics committee of Shenzhen Kangning Hospital. Written informed consent will be obtained from the participants after detailed explanations of the purpose and procedures of the trial have been provided.

\section{Consent for publication}

Not applicable.

\section{Competing interests}

The authors declare that they have no competing interests.

\section{Author details}

${ }^{1}$ Department of Psychiatry, Shenzhen Kangning Hospital, Shenzhen, Guangdong, China. ${ }^{2}$ Affiliated Shenzhen Clinical College of Psychiatry, Jining Medical University, Jining, Shandong, China. ${ }^{3}$ Center of Acute Psychiatry Service, Second People's Hospital of Huizhou, Huizhou, Guangdong, China. ${ }^{4}$ Department of Biostatistics, School of Public Health, Southern Medical University, Guangzhou, China. ${ }^{5}$ Laboratory of Brain Stimulation and Biological Psychiatry, Brain Function and Psychosomatic Medicine Institute, Second People's Hospital of Huizhou, Huizhou, Guangdong, China. ${ }^{6}$ CAS Key Laboratory of Mental Health, Institute of Psychology, Chinese Academy of Sciences, Beijing, China. ${ }^{7}$ Key Laboratory of Intelligent Information Processing, Advanced Computer Research Center, Institute of Computing Technology, Chinese Academy of Sciences, Beijing, China.

Received: 5 May 2019 Accepted: 11 October 2019 Published online: 06 November 2019

\section{References}

1. Disease GBD, Injury I, Prevalence C. Global, regional, and national incidence, prevalence, and years lived with disability for 328 diseases and injuries for 195 countries, 1990-2016: a systematic analysis for the global burden of Disease study 2016. Lancet. 2017;390(10100):1211-59.

2. Dong M, Wang SB, Li Y, Xu DD, Ungvari GS, Ng CH, Chow IHI, Xiang YT. Prevalence of suicidal behaviors in patients with major depressive disorder in China: a comprehensive meta-analysis. J Affect Disord. 2018;225:32-9.

3. Kupferberg A, Bicks L, Hasler G. Social functioning in major depressive disorder. Neurosci Biobehav Rev. 2016;69:313-32.

4. MacQueen GM, Memedovich KA. Cognitive dysfunction in major depression and bipolar disorder: assessment and treatment options. Psychiatry Clin Neurosci. 2017;71(1):18-27.

5. Phillips MR, Li X, Zhang Y. Suicide rates in China, 1995-99. Lancet. 2002; 359(9309):835-40.

6. Milev RV, Giacobbe P, Kennedy SH, Blumberger DM, Daskalakis ZJ, Downar J, Modirrousta M, Patry S, Vila-Rodriguez F, Lam RW, et al. Canadian network for mood and anxiety treatments (CANMAT) 2016 clinical guidelines for the Management of Adults with major depressive disorder: section 4. Neurostimulation Treatments. Can J Psychiatr. 2016;61(9):561-75.

7. Fink M, Taylor MA. Electroconvulsive therapy: evidence and challenges. JAMA. 2007;298(3):330-2.

8. Rasmussen K. The practice of electroconvulsive therapy: recommendations for treatment, training, and privileging (2nd edition). J ECT. 2002;18(1):58-9.

9. Wang ZM, Zhu H, Pan YL, Chiu HF, Correll CU, Ungvari GS, Lai KY, Cao XL, Li $Y$, Zhong BL, et al. Electroconvulsive therapy and its association with demographic and clinical characteristics in Chinese psychiatric patients. J ECT. 2015;31(2):114-8.

10. Sienaert P, Vansteelandt K, Demyttenaere K, Peuskens J. Randomized comparison of ultra-brief bifrontal and unilateral electroconvulsive therapy for major depression: cognitive side-effects. J Affect Disord. 2010;122(1-2):60-7.

11. Cusin C, Dougherty DD. Somatic therapies for treatment-resistant depression: ECT, TMS, VNS, DBS. Biol Mood Anxiety Disord. 2012;2:14.

12. Mankad MV, Beyer JL, Weiner RD, Krystal A. Clinical manual of electroconvulsive therapy: American psychiatric pub; 2010.

13. Kellner CH, Knapp R, Husain MM, Rasmussen K, Sampson S, Cullum M, McClintock SM, Tobias KG, Martino C, Mueller M, et al. Bifrontal, bitemporal and right unilateral electrode placement in ECT: randomised trial. $\mathrm{Br}$ J Psychiatry. 2010;196(3):226-34.

14. Tran DV, Meyer JP, Farber KG, Chen XR, Rosenthal BD, Kellner CH. Rapid response to electroconvulsive therapy: a case report and literature review. J ECT. 2017;33(3):e20-1.

15. Lapidus KA, Shin JS, Pasculli RM, Briggs MC, Popeo DM, Kellner CH. Lowdose right unilateral electroconvulsive therapy (ECT): effectiveness of the first treatment. J ECT. 2013;29(2):83-5.

16. Rich CL. Recovery from depression after one ECT. Am J Psychiatry. 1984; 141(8):1010-1. 
17. Association AP: The practice of electroconvulsive therapy: recommendations for treatment, training, and privileging (a task force report of the American Psychiatric Association): American psychiatric pub; 2008.

18. Prudic J, Sackeim HA, Devanand DP, Krueger RB, Settembrino JM. Acute cognitive effects of subconvulsive electrical stimulation. Convuls Ther. 1994; 10(1):4-24.

19. Beran M, Perkins JC, Scollon RW. Psychological studies on patients undergoing nonconvulsive electric-stimulation treatment. Am J Psychiatry. 1952;109(5):367-74.

20. Alexander L. Nonconvulsive electric stimulation therapy; its place in the treatment of affective disorders, with notes on the reciprocal relationship of anxiety and depression. Am J Psychiatry. 1950;107(4):241-50.

21. Regenold WT, Noorani RJ, Piez D, Patel P. Nonconvulsive electrotherapy for treatment resistant unipolar and bipolar major depressive disorder: a proofof-concept trial. Brain Stimul. 2015;8(5):855-61.

22. Li MZ, Chen LC, Rong H, Xu SX, Li Y, Yang QF, Deng WF, Yang HZ, Kong XM, Xiao L, et al. Low-charge electrotherapy for patients with schizophrenia: a double-blind, randomised controlled pilot clinical trial. Psychiatry Res. 2018;272:676-81.

23. Organization $\mathbf{W H}$. The ICD-10 classification of mental and behavioural disorders: diagnostic criteria for research. Geneva: World Health Organization; 1993.

24. Wechsler D. Wechsler adult intelligence scale-fourth edition (WAIS-IV). San Antonio: The Psychological Corporation; 2008.

25. Young RC, Biggs JT, Ziegler VE, Meyer DA. A rating scale for mania: reliability, validity and sensitivity. Br J Psychiatry. 1978;133:429-35.

26. Guy W. ECDEU assessment manual for psychopharmacology. Rockville: US Department of Health, and Welfare; 1976. p. 534-7.

27. Kay SR, Fiszbein A, Opler LA. The positive and negative syndrome scale (PANSS) for schizophrenia. Schizophr Bull. 1987;13(2):261-76.

28. Ware JE Jr, Sherbourne CD. The MOS 36-item short-form health survey (SF36): I. Conceptual framework and item selection. Med Care. 1992;30:473-83.

29. Hamilton M. The assessment of anxiety states by rating. Br J Med Psychol. 1959;32(1):50-5.

30. Zung WW. A self-rating depression scale. Arch Gen Psychiatry. 1965;12(1): 63-70.

31. Zung WW. A rating instrument for anxiety disorders. Psychosomatics. 1971 12(6):371.

32. Sunderland A, Harris JE, Baddeley AD. Do laboratory tests predict everyday memory? A neuropsychological study. J Mem Lang. 1983;22(3):341.

33. Pangman VC, Sloan J, Guse L. An examination of psychometric properties of the mini-mental state examination and the standardized mini-mental state examination: implications for clinical practice. Appl Nurs Res. 2000;13(4):209-13.

34. Randolph C, Tierney MC, Mohr E, Chase TN. The repeatable battery for the assessment of neuropsychological status (RBANS): preliminary clinical validity. J Clin Exp Neuropsychol. 1998;20(3):310-9.

35. Dickinson D, Ramsey ME, Gold JM. Overlooking the obvious: a meta-analytic comparison of digit symbol coding tasks and other cognitive measures in schizophrenia. Arch Gen Psychiatry. 2007;64(5):532-42.

36. Stroop JR. Studies of interference in serial verbal reactions. J Exp Psychol Gen. 1992;121(1):15.

37. Rugh $\mathrm{R}$. The effect of the adult anterior pituitary hormone on the tadpoles and the immature male frogs of the bullfrog, Rana Catesbiana. Biol Bull. 1946;90(3):291-300.

38. Sobin C, Sackeim HA, Prudic J, Devanand DP, Moody BJ, McElhiney MC. Predictors of retrograde amnesia following ECT. Am J Psychiatry. 1995; 152(7):995-1001.

39. Youn JC, Kim KW, Lee DY, Jhoo JH, Lee SB, Park JH, Choi EA, Choe JY, Jeong JW, Choo $\mathrm{H}$. Development of the subjective memory complaints questionnaire. Dement Geriatr Cogn Disord. 2009;27(4):310-7.

40. Kong XM, Xu SX, Sun Y, Wang KY, Wang C, Zhang J, Xia JX, Zhang L, Tan BJ, $\mathrm{Xie} \mathrm{XH}$. Electroconvulsive therapy changes the regional resting state function measured by regional homogeneity (ReHo) and amplitude of low frequency fluctuations (ALFF) in elderly major depressive disorder patients: an exploratory study. Psychiatry Res Neuroimaging. 2017;264:13-21.

41. Jorgensen A, Magnusson P, Hanson LG, Kirkegaard T, Benveniste H, Lee H, Svarer C, Mikkelsen JD, Fink-Jensen A, Knudsen GM, et al. Regional brain volumes, diffusivity, and metabolite changes after electroconvulsive therapy for severe depression. Acta Psychiatr Scand. 2016;133(2):154-64.
42. Sartorius A, Demirakca T, Bohringer A, Clemm von Hohenberg C, Aksay SS, Bumb JM, Kranaster L, Ende G. Electroconvulsive therapy increases temporal gray matter volume and cortical thickness. Eur Neuropsychopharmacol. 2016;26(3):506-17.

43. Nuninga JO, Mandl RCW, Boks MP, Bakker S, Somers M, Heringa SM, Nieuwdorp W, Hoogduin H, Kahn RS, Luijten P, et al. Volume increase in the dentate gyrus after electroconvulsive therapy in depressed patients as measured with 7T. Mol Psychiatry. 2019. [Epub ahead of print].

44. Drago T, O'Regan PW, Welaratne I, Rooney S, O'Callaghan A, Malkit M, Roman E, Levins KJ, Alexander L, Barry D, et al. A comprehensive regional neurochemical theory in depression: a protocol for the systematic review and meta-analysis of $1 \mathrm{H}-\mathrm{MRS}$ studies in major depressive disorder. Syst Rev. 2018;7(1):158.

45. Takei Y, Fujihara K, Tagawa M, Hironaga N, Near J, Kasagi M, Takahashi Y, Motegi T, Suzuki Y, Aoyama Y, et al. The inhibition/excitation ratio related to task-induced oscillatory modulations during a working memory task: a multtimodal-imaging study using MEG and MRS. Neuroimage. 2016;128: 302-15.

46. Xi G, Hui J, Zhang Z, Liu S, Zhang X, Teng G, Chan KC, Wu EX, Nie B, Shan B, et al. Learning and memory alterations are associated with hippocampal Nacetylaspartate in a rat model of depression as measured by $1 \mathrm{H}-\mathrm{MRS}$. PLoS One. 2011:6(12):e28686.

47. Oostenveld R, Praamstra P. The five percent electrode system for highresolution EEG and ERP measurements. Clin Neurophysiol. 2001;112(4): 713-9.

48. Zhou L, Wang G, Wang H. Abnormalities of P300 before and after antidepressant treatment in depression: an ERP-sLORETA study. Neuroreport. 2018;29(3):160-8.

49. Kutas M, McCarthy G, Donchin E. Augmenting mental chronometry: the P300 as a measure of stimulus evaluation time. Science. 1977;197(4305): $792-5$.

50. Kuang W, Tian L, Yue L, Li J. Effects of escitalopram with a Chinese traditional compound Jiuweizhenxin-keli on mismatch negativity and P50 in patients with major depressive disorders. Neuropsychiatr Dis Treat. 2016;12: 1935-41.

51. Arnfred SM, Chen AC, Glenthøj BY, Hemmingsen RP. Normal p50 gating in unmedicated schizophrenia outpatients. Am J Psychiatr. 2003;160(12): 2236-8.

52. Hutchison AK, Hunter SK, Wagner BD, Calvin EA, Zerbe GO, Ross RG. Diminished infant P50 sensory gating predicts increased 40-month-old attention, anxiety/depression, and externalizing symptoms. J Atten Disord. 2017;21(3):209-18.

53. Julious SA. Sample sizes for clinical trials with normal data. Stat Med. 2004 23(12):1921-86

54. Fischl B. FreeSurfer. Neuroimage. 2012;62(2):774-81.

55. Jenkinson M, Beckmann CF, Behrens TE, Woolrich MW, Smith SM. Fsl. Neuroimage. 2012;62(2):782-90.

56. Kaatiala J, Yrttiaho S, Forssman L, Perdue K, Leppanen J. A graphical user interface for infant ERP analysis. Behav Res Methods. 2014;46(3):745-57.

\section{Publisher's Note}

Springer Nature remains neutral with regard to jurisdictional claims in published maps and institutional affiliations.

Ready to submit your research? Choose BMC and benefit from:

- fast, convenient online submission

- thorough peer review by experienced researchers in your field

- rapid publication on acceptance

- support for research data, including large and complex data types

- gold Open Access which fosters wider collaboration and increased citations

- maximum visibility for your research: over $100 \mathrm{M}$ website views per year

At $\mathrm{BMC}$, research is always in progress.

Learn more biomedcentral.com/submissions 